\title{
Proposal on the Diagnostic Criteria of Definite Isolated Otolith Dysfunction
}

\author{
Kenneth Wei De Chua ${ }^{1,2}$, Heng Wai Yuen ${ }^{1}$, David Andrew Yong Ming Low ${ }^{1}$, and Savitha Kamath ${ }^{1}$ \\ ${ }^{1}$ Department of Otolaryngology, Audiology, Changi General Hospital, Singapore \\ ${ }^{2}$ The American Institute of Balance, Largo, FL, USA
}

\section{Dear Editor,}

We read the article written by Myung-Whan Suh and colleagues [1] entitled "Proposal on the diagnostic criteria of definite isolated otolith dysfunction (iOD)" with great interest. In this study, the authors proposed a diagnostic criterion for iOD. Thank you for highlighting this under-represented clinical presentation, which is not uncommonly seen. Nevertheless, we wish to present some thoughts for your consideration.

We think that primary idiopathic definite/probably iOD is easier to consider when there is no clear clinical diagnosis and patients present with symptoms of otolith dysfunction (rocking, swaying, tilting, disequilibrium, floating etc $\cdots$ ) and/ or with laboratory-based confirmation. One of the arguments here could be that the pathognomonic otolith symptoms are not well understood yet and may be easily confused with descriptors of non-specific dizziness, which are not coming from the otoliths. Nevertheless, when clinical diagnosis is unclear, it is tempting to consider iOD as an emerging clinical entity.

However, when considering secondary iOD with known or suspected vestibular diagnosis, it may be more challenging especially for fluctuating conditions such as Meniere's disease (MD) and vestibular migraine (VM). In addition, the added confounding variable of central compensation may result in "false negative" laboratory test results as the authors have pointed out.

Received October 5, 2020 / Accepted October 27, 2020

\section{Address for correspondence}

Kenneth Wei De Chua, M.Sc FAIB

Department of Otolaryngology, Audiology, Changi General Hospital, 2 Simei St 3, Singapore 529889, Singapore

Tel +65-6-9365259/E-mail kenneth_chua@cgh.com.sg

This is an Open Access article distributed under the terms of the Creative Commons Attribution Non-Commercial License (https://creativecommons.org/licenses/by-nc/4.0/) which permits unrestricted non-commercial use, distribution, and reproduction in any medium, provided the original work is properly cited.
For example in MD, there may be a sequential progressive lesion of the labyrinthine that eventually includes other structures, although the otoliths have been reported to be more sensitive than semi-circular canals to hydropic expansion [2]. If laboratory test suggests that the otoliths are the only structures affected, it does not rule out the involvement of other structures. As MD progresses and fluctuates, the patients may find themselves in a dynamic state of compensation-decompensation with variable laboratory test results. In fact, some authors are suggesting that the discordant video-head impulse test (normal) with calorics (abnormal) happens in $60-70 \%$ of patients with MD [3] and hence may not fit the clinical picture of iOD.

If we look at VM as a differential diagnosis due to the overlapping symptoms with MD, we are also considering that migraine is a neurovascular event that undeniably involves central pathways. The stability of vestibular evoked myogenic potential (VEMP) latencies may argue against a central pathology but does not rule out non-destructive changes in brainstem nuclear sensitivity [4]. Hence, abnormal VEMPs may not always suggest a peripheral pathology.

Where Benign Paroxysmal Positional Vertigo (BPPV) is concerned, although there is a correlation between BPPV and otolith dysfunction with degeneration or denervation of the utricle leading to otoconia detachment [5], the relationship is neither causal nor coincidental. Hence, we agree with the authors' focus on definite versus probable iOD instead of whether iOD is of a primary idiopathic cause or secondary to known vestibulopathy.

Finally, the management plans for iOD remains elusive as there are no current rehabilitation techniques for improving otolith function with the exception of adaptation of vestibular spinal reflexes. Nevertheless, continuous and further refinement of iOD diagnosis will lay the groundwork for the eventual conceptualisation and development of rehabilitation strategies. Thank you for the interesting article and we hope to be able 
to validate your findings in our own clinical studies.

\section{Acknowledgments}

None

\section{Conflicts of interest}

The authors have no financial conflicts of interest.

\section{ORCID iDs}

Kenneth Wei De Chua https://orcid.org/0000-0002-2677-1183

Heng Wai Yuen https://orcid.org/0000-0001-7510-2405

David Andrew Yong Ming Low

https://orcid.org/0000-0002-6302-8364

Savitha Kamath https://orcid.org/0000-0003-2945-9985

\section{REFERENCES}

1) Park HG, Lee JH, Oh SH, Park MK, Suh MW. Proposal on the diagnostic criteria of definite isolated otolith dysfunction. J Audiol Otol 2019;23:103-11.

2) Pender DJ. Membrane stress in the human labyrinth and meniere disease: a model analysis. Int Arch Otorhinolaryngol 2015;19:336-42.

3) Hannigan IP, Welgampola MS, Watson SRD. Dissociation of caloric and head impulse tests: a marker of Meniere's disease. J Neurol 2019. http://doi.org/10.1007/s00415-019-09431-9.

4) Zuniga MG, Janky KL, Schubert MC, Carey JP. Can vestibularevoked myogenic potentials help differentiate Ménière disease from vestibular migraine? Otolaryngol Head Neck Surg 2012;146:788-96.

5) Lee SK, Kim SJ, Park MS, Byun JY. Otolith organ function according to subtype of benign paroxysmal positional vertigo. Laryngoscope 2014;124:984-8. 